\title{
RECURRENCE IN MALE BREAST CANCER: A CASE SERIES
}

Rafael Everton Assunção Ribeiro da Costaํ, Fergus Tomás Rocha de Oliveira', Eduarda Norberto Siqueira', Ana Lúcia Nascimento Araújo², Sabas Carlos Vieira ${ }^{3}$

${ }^{1}$ Universidade Estadual do Piauí - Teresina (PI), Brazil.

${ }^{2}$ Hospital São Marcos - Teresina (PI), Brazil.

${ }^{3}$ Oncocenter - Teresina (PI), Brazil.

Introduction: Male breast cancer (MBC) is a very rare disease, which accounts for approximately $1.0 \%$ of all breast cancers and around $0.17 \%-1.0 \%$ of all male malignancies, more common in the age group of 60 years, and $93.7 \%$ of cases of $\mathrm{MBC}$ are histologically classified as the invasive carcinoma of no special type (ICNS). Treatment (i.e., surgery, radiotherapy, chemotherapy, and hormone therapy) and prognosis are similar to breast cancer in women. The aim of this study was to describe five MBC cases and to analyze the occurrence of relapse in a follow-up of 88 months (36-180 months). Case series: The mean age of five patients of the case series was 69 years (57-88 years). All cases were histologically classified as ICNS. The mean tumor size of the series was $2.6 \mathrm{~cm}(1.0-5.2 \mathrm{~cm})$. One patient of the case series presented with perineural invasion, and another case had axillary node involvement $(+5 / 15)$. None of the patients had metastasis at the time of diagnosis. All patients underwent surgery. Regarding complementary treatment, one patient underwent chemotherapy, radiotherapy, and hormone therapy (i.e., a case with axillary node involvement); two others underwent chemotherapy and hormone therapy; and the remaining two cases underwent treatment only with hormone therapy. One patient had systemic recurrence with the presentation of bone metastasis ( 75 months of follow-up). The study was approved by a Research Ethics Committee, under CAAE No 30154720.0.0000.5209. Conclusion: With a mean follow-up of 88 months (36-180 months), one of the five patients in the series (20\%) showed systemic recurrence with bone metastasis.

Keywords: Recurrence; Breast Neoplasms; Male; Medical Records. 